J3eA, Journal sur l'enseignement des sciences et technologies de l'information et des systèmes, Volume 4, Hors-Série 1, 4 (2005)

DOI : http://dx.doi.org/10.1051/bib-j3ea:2005604

(C) EDP Sciences, 2005

Influence des charges non linéaires dans les réseaux de bord : application aux réseaux d'avion

F. Barruel, J-L. Schanen, N. Retiere et J. Roudet

Laboratoire d'Électrotechnique de Grenoble (LEG)

CNRS UMR5529 INPG/UJF - ENSIEG

BP 46

F-38402 SMH CEDEX, Grenoble, France 


\title{
Influence des charges non linéaires dans les réseaux de bord: application aux réseaux d'avion
}

\author{
F. Barruel, J-L. Schanen, N. Retiere, J. Roudet
}

Laboratoire d'Electrotechnique de Grenoble - LEG -CNRS UMR5529 INPG/UJF - ENSIEG, BP.46, 38402 SMH Cedex, Grenoble France

Résumé- Cet article a pour but d'illustrer un des aspects du projet intégrateur proposé aux étudiants du nouveau master professionnel "csee" (conception des systèmes d'énergie électrique) à Grenoble. Il porte sur le dimensionnement de filtres passifs pour les convertisseurs d'électronique de puissance. D'ordinaire, on suppose la génération d'harmonique indépendante du filtre. Ceci n'est pas le cas avec les convertisseurs d'électronique de puissance, dont le régime de fonctionnement -et donc les perturbations- dépend des paramètres du réseau -et donc du filtre. Par ailleurs, les réseaux embarqués de l'avion du futur, s'ils seront peut-être encore alternatifs, ne seront plus à fréquence fixe... Le dimensionnement d'un filtre passif sous toutes ces conditions devient alors un véritable casse-tête, et seule une approche globale, utilisant des moyens de simulation, permet une appréhension complète du problème. Le projet est illustré par l'exemple d'un redresseur triphasé à diodes, sur charge capacitive, représentatif de la majorité des étages d'entrée des actionneurs électriques ou électrohydrauliques utilisés dans l'aéronautique.

\section{LE MASTER "CSEE" ET SON PROJET INTEGRATEUR}

Le projet présenté dans cet article est proposé aux étudiants $\mathrm{du}$ tout récent master professionnel "csee" (conception des systèmes d'énergie électrique) à Grenoble. Avant de rentrer dans la description du projet lui-même, nous nous proposons de présenter la formation en elle-même.

\section{A. Contexte métier}

Depuis maintenant plusieurs décennies, le génie électrique gagne du terrain dans tous les domaines industriels ou domestiques. Cette tendance n'a fait que se renforcer depuis quelques années grâce aux progrès de l'électronique de puissance qui ont été considérables dans la maîtrise de la conversion et de la distribution de l'énergie électrique.

Ces progrès ont d'abord concerné les procédés d'alimentation des systèmes électriques: machines outils, traction ferroviaire, systèmes informatiques, électroménager,... En effet, l'utilisation systématique de convertisseurs d'électronique de puissance permet un nombre important de fonctions jusqu'alors impensables: rendement très élevé, fiabilité, disponibilité et continuité de service, compacité et légèreté, discrétion acoustique et électromagnétique (CEM),...

La multiplication de ces dispositifs pose toutefois le problème plus large $\mathrm{du}$ fonctionnement et $\mathrm{du}$ dimensionnement du réseau électrique qui les interconnecte: quelles sources d'énergie et moyens de stockage, quelles architectures de réseau, quelles stratégies de protection, quels moyens de communication entre les différents composants, quelle fiabilité attendue ...?

De ce fait nous assistons à une évolution des métiers du génie électrique qui nécessitent de nouvelles compétences autour de:

- La capacité de mener une réflexion de type "système",

- L'intégration des contraintes technologiques propres au couplage d'un composant sur un réseau,

- La maîtrise des outils de simulation et prototypage virtuel en vue de l'analyse et de la conception de ces réseaux.

\section{B. Contexte formation}

Les formations dispensées dans le domaine du génie électrique sur le site Grenoblois ont toujours été en adéquation avec l'évolution des métiers. C'est particulièrement vrai pour l'ENSIEG (école nationale supérieure des ingénieurs électriciens de grenoble) qui conduit à une formation d'ingénieurs électriciens polyvalents, capables d'assumer un rôle majeur et de hautes responsabilités au sein d'entreprises dans de très nombreux secteurs. Pourtant, le nouveau contexte industriel nécessite d'enrichir cette offre de formation en proposant un parcours plus technologique et applicatif. Ce parcours est plus naturellement destiné à des étudiants ayant déjà suivi un enseignement à caractère technologique.

\section{Objectif de la formation}

Au travers de cette formation l'étudiant s'intéressera à la conception de plusieurs types de petits réseaux (réseaux de bord d'avions, de voitures, de navires, de bâtiments, d'usines, ferroviaires,...). Plusieurs aspects seront abordés comme

- Les composants constituant les réseaux (convertisseurs d'électronique de puissance, alternateurs, câbles de puissance, capteurs,...)

- les grandes fonctions nécessaires à la bonne marche d'un réseau (contrôle commande, filtrage, protection,...)

- les méthodes de modélisation, d'analyse et de conception.

\section{Organisation de la formation}

La formation repose sur deux grands principes,

- un projet transversal intégrateur des connaissances et des compétences nécessaires à la conception des systèmes d'énergie électriques (150h de BE),

- des modules d'enseignement choisis parmi ceux proposés à l'ENSIEG en accord avec les objectifs de la formation (250 $\mathrm{h}$ de cours/TD/BE) 


\section{E. Le projet intégrateur}

Ce projet se décompose en 5 modules. L'objectif est de fournir une méthodologie générale qui permette de s'adapter à la conception et au dimensionnement de tout type de réseau. De ce fait, le réseau de bord d'avion est un exemple parmi d'autres, qui permet de mettre ces connaissances en pratique.

- Module 1: dimensionnement usuel d'un système électrique (14hCM - 28hBE)

- Module 2: pré-dimensionnement statique (6hCM - 20hBE)

- Module 3: dimensionnement dynamique (6hCM-16hBE)

- Module 4 : dimensionnement harmonique (6hCM-20hBE)

- Module 5 : intégration et validation finale (20hBE)

C'est le module 4 qui est développé dans cet article, sur l'exemple d'un réseau de bord d'avion, dont les contraintes sont données au paragraphe suivant. Ce module comporte une séance de $2 \mathrm{~h} 00$ de présentation, et 6 séances de $4 \mathrm{~h} 00$ de BE. Des temps de CM sont intégrés aux séances quand cela est nécessaire.

\section{LES CONTRAINTES DU RESEAU DE BORD "AVION"}

L'aéronautique est en train de vivre la même mutation que l'automobile il y a quelques années, avec la mise en œuvre du concept de "plus électrique" [1]. D’un coté, l'énergie utilisée pour le confort du passager augmente: on compte actuellement $12 \mathrm{~kW} /$ passager, et on table sur $30 \mathrm{~kW}$ à 3 ans (avec les prises pour PC portables), et $50 \mathrm{~kW}$ après 2010 (jeux vidéos...). Par ailleurs, le désir d'améliorer les rendements conduit à envisager sérieusement l'abandon de la distribution hydraulique, au profit d'actionneurs électohydrauliques, voire électriques. L'objectif est de fournir la puissance électrique supplémentaire nécessaire tout en garantissant une meilleure fiabilité et sûreté de fonctionnement, une amélioration du rendement global ainsi qu'une plus grande souplesse. Cette étude fait l'objet du contrat européen "Power Optimised Aircraft" [2], dont la vocation est de tester les nouvelles technologies, à la fois en simulation et en grandeur réelle.

Comme l'automobile, donc ?... Au détail près que les contraintes de fiabilité sont légèrement différentes ! Par ailleurs, le réseau automobile, hormis sa mutation annoncée à plus ou moins court terme vers le $42 \mathrm{~V}$, ne subira pas la même modification radicale que celle qui est envisagée pour l'avion, dont le réseau est, de plus, singulièrement plus complexe (plusieurs générateurs, protections....).

La première modification notable sur l'A380, futur gros porteur de Airbus, est la suppression de l'embrayage électromécanique qui assurait jusqu'à présent une vitesse constante sur l'arbre de l'alternateur. Ce réseau à fréquence variable introduit automatiquement une augmentation des convertisseurs électriques. Cette tendance est renforcée par l'abandon des actionneurs hydrauliques, à la maintenance lourde et à la souplesse de fonctionnement limitée, au profit d'actionneurs électriques dont l'alimentation se fait nécessairement par des convertisseurs statiques.

La prolifération de ces organes de conversion, non linéaires, a pour conséquence la prolifération d'harmoniques sur le réseau embarqué [3]. Comment réagira l'alternateur dans cette configuration? Comment se comporteront les autres charges? Les pertes augmenteront-elle d'une manière significative ? Pour le moment, les avionneurs répondent à ces interrogations en conservant les normes actuelles en terme de Taux de Distorsion Harmonique (THD $<5 \%-10 \%$ ), et de limitations rang par rang, comme indiqué dans le Tableau 1 ci-dessous. Il est donc nécessaire de munir les équipements de conversion de filtres adaptés.

Or le dimensionnement de filtres passifs pour convertisseurs statiques n'est pas si aisé quand on cherche à le systématiser. En effet, contrairement à l'approche "classique" utilisée en filtrage, la source de perturbation dépend des impédances du réseau, c'est-à-dire entre autre du filtre ! Ceci sera montré au paragraphe V.

\begin{tabular}{c|c}
\hline Harmoniques & Limites \\
\hline 3,5 et 7 & $\mathrm{I} 3=\mathrm{I} 5=\mathrm{I} 7=0.02 \mathrm{I} 1$ \\
\hline $\mathrm{n}=9,15,21, \ldots, 39$ & $\mathrm{In}=0.1 \mathrm{I} 1 / \mathrm{n}$ \\
\hline 11 & $\mathrm{I} 11=0.1 \mathrm{I} 1$ \\
\hline 13 & $\mathrm{I} 13=0.08 \mathrm{I} 1$ \\
\hline 17 et 19 & $\mathrm{I} 17=\mathrm{I} 19=0.04 \mathrm{I} 1$ \\
\hline 23 et 25 & $\mathrm{I} 23=\mathrm{I} 25=0.03 \mathrm{I} 1$ \\
\hline $29,31,35$ et 37 & $\mathrm{In}=0.3 \mathrm{I} 1 / \mathrm{n}$ \\
\hline
\end{tabular}

TABLEAU 1 : NORME AIRBUS ABD0100 POUR LES SYSTEMES TRIPHASES

\section{UN ETAGE D'ENTREE "TYPE" D'UN ACTIONNEUR}

Tout actionneur électrique moderne est alimenté par un onduleur de type MLI. Celui-ci nécessite un bus continu, qui pour des raisons de coût et de simplicité est réalisé selon la structure pont de diodes sur capacité en tête, décrit Fig. 1.

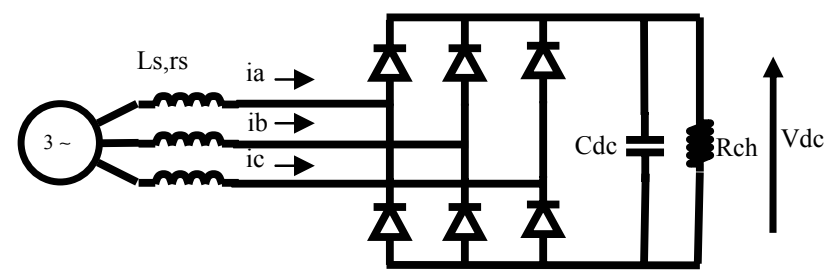

Fig. 1. Redresseur triphasé à diodes sur capacité en tête

On représente l'ensemble "onduleur MLI - actionneur charge" par une résistance équivalente destinée à absorber la même puissance active.

Cette structure ne peut fonctionner, en terme d'électronique de puissance, que grâce à la présence des inductances de ligne $L s \ldots$

Le premier travail est de comprendre le fonctionnement de ce type de montage. Pour cela le logiciel Pspice est utilisé. Il permet de simuler aisément ce redresseur en temporel, grâce à la FFT intégrée et d'analyser le contenu harmonique des courant ligne. Un exemple est donné Fig. 2.

Les étudiants sont invités à analyser l'influence des divers paramètres de ce montage, et notamment le rôle des inductances de ligne, sur le contenu harmonique absorbé. On notera que la tension de sortie Vdc (et donc la puissance absorbée dans la modélisation adoptée) est également affectée par cette modification. Il conviendra donc de la réajuster après toute modification de $L s$, de manière à conserver la puissance constante. De plus, on quantifiera l'effet de ces variations d'inductance de ligne sur l'énergie 
réactive absorbée (facteur de puissance).

Afin de pouvoir aisément effectuer les calculs comme le taux de distorsion harmonique (THD), le facteur de puissance, et par exemple superposer le spectre basse fréquence à la norme, on exporte les courbes de Spice vers une feuille de calcul Mathcad (copier-coller de Spice vers un fichier texte, et fonction LIREPRN de Mathcad).

Ceci constitue la première séance.
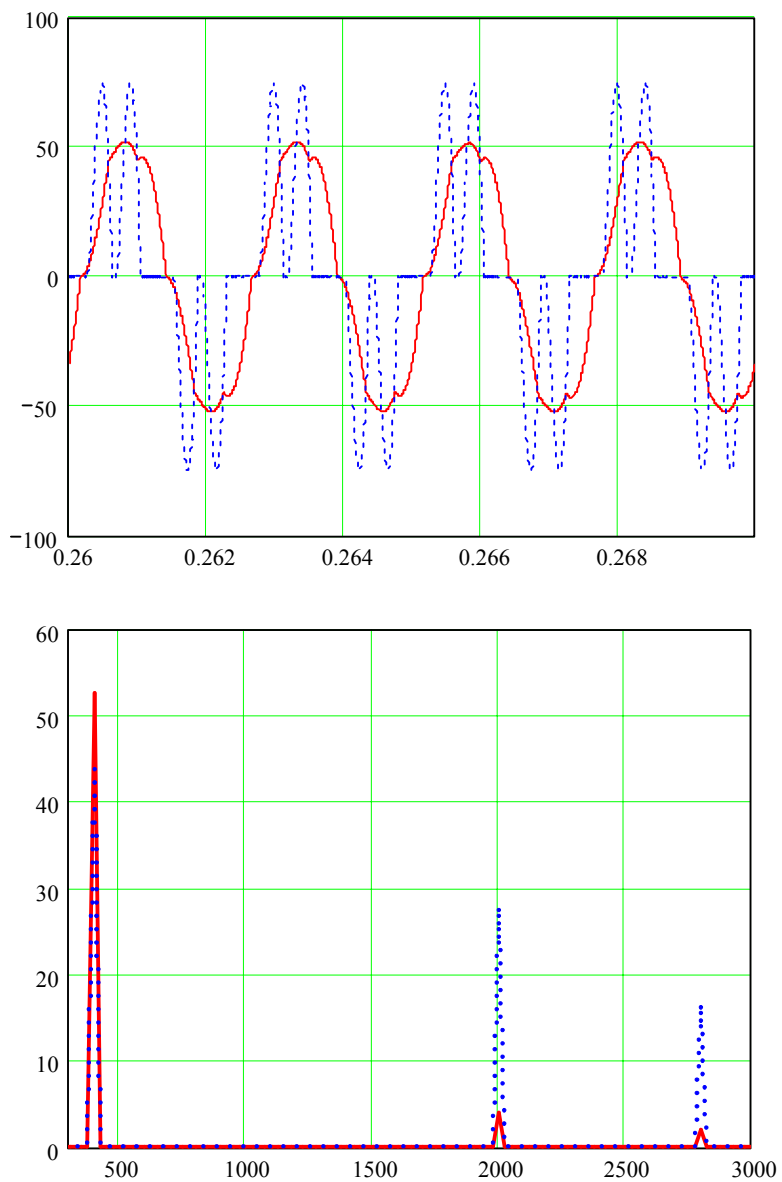

Fig. 2. Exemple de forme d'onde du courant absorbé pour $\mathrm{La}=518 \mu \mathrm{H}$ (traits pleins) et $10 \mu \mathrm{H}$ (pointillés), $\mathrm{R}=4.1 \Omega$ (traits pleins) et $\mathrm{R}=6.7 \Omega$ (pointillés) - pour conserver une puissance de $10 \mathrm{~kW}$ dans les deux cas-, une tension d'alimentation de $115 \mathrm{Veff}, 400 \mathrm{~Hz}$.

Au dessous, analyse harmonique (FFT Spice) pour les deux cas.

\section{LES STRUCTURES DE FILTRAGE PASSIF CLASSIQUES}

\section{A. Les structures}

La première séance a montré que les normes aéronautiques ne sont pas respectées par ce montage, à moins d'augmenter les inductances de ligne à un tel point que le facteur de puissance est trop fortement dégradé. Il convient donc de mettre en œuvre une solution de filtrage. Plusieurs solutions de filtres passifs parallèles sont possibles, comme indiqué Fig. 3. Ils permettent de limiter la consommation de puissance réactive et/ou le taux de réjection harmonique sur le réseau. On retrouve généralement quatre types [4] [5] [6]:

- L'inductance de ligne (dite de lissage) qui permet d'atténuer l'ensemble du spectre.

- Les filtres résonants, le plus souvent utilisés pour éliminer des harmoniques précises ( 3 ou 5 en monophasé ou triphasé).
- Les filtres passe-bas, d'ordre variable, permettent d'atténuer un ensemble d'harmoniques mais ne possèdent pas un très bon facteur de qualité.

- Les filtres double passe-bande, permettent de filtrer deux rangs en même temps.

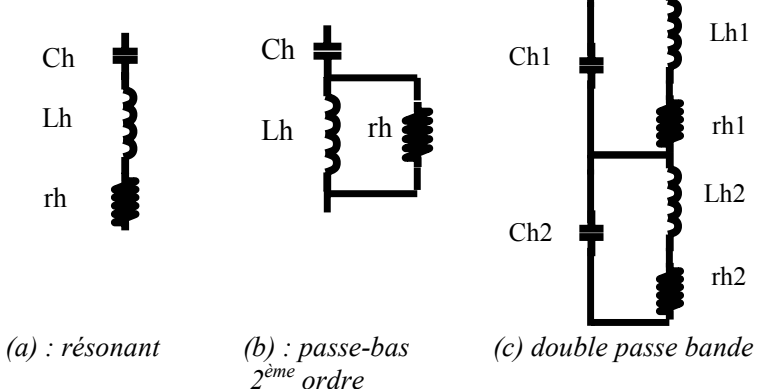

Fig. 3. Plusieurs structures de filtre passif.

Nous proposons de comparer ces filtres, à l'aide du logiciel Mathcad. L'objectif commun de ces filtres est d'avoir à la fois une faible impédance aux fréquences polluantes et une impédance élevée au fondamental. Pour calculer la fonction de transfert de ces filtres, on utilise la notion de quadripôle (Fig. 4). X, Y et $\mathrm{Z}$ représentent les impédances complexes des branches du filtre [7]. De plus, cette notation facilite le calcul de la matrice chaîne en présence de plusieurs filtres en cascade.

La fonction de transfert intéressante pour notre étude est I1/I2, si le convertisseur est branché côté 2 , et le réseau se situe côté 1. La Fig. 5 montre que l'atténuation apportée par une inductance seule est nulle, mais qu'elle renforce nettement l'effet des filtres parallèles. On constate également immédiatement le risque d'amplification du courant côté réseau: il faut que ce pic de résonance ne corresponde à aucun signal présent sur le réseau (harmonique, fréquence de communication...).

Nous choisissons le filtre résonant (a- Fig. 3) et l'inductance série de ligne pour la suite de l'étude. Dans un soucis de simplicité (et de minimisation du nombre de composants), on se limitera à un seul filtre.

La méthodologie de dimensionnement du filtre résonant est exposée dans les parties suivantes. Elle occupe une séance.

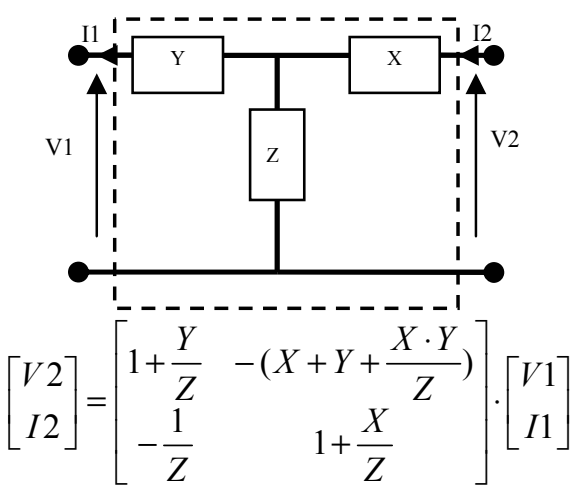

Fig. 4. Représentation d'un quadripôle en $\mathrm{T}$ et sa fonction de transfert sous forme matricielle

\section{B. Dimensionnement de l'inductance série seule}

Avant de dimensionner les composants du filtre, il est indispensable de fixer la valeur de l'inductance série. En utilisant la simulation Spice et les exploitation sous Mathcad 
précédentes, on peut tracer les courbes de la Fig. 6 (ou tout au moins quelques points). On voit pour une variation de Ls de $100 \mu \mathrm{H}$ à $800 \mu \mathrm{H}$ que le $\mathrm{TDH}$ passe de 30 à $5 \%$. Cependant, ce n'est pas sans conséquences sur le facteur de puissance (FP) qui atteint 0.65. L'utilisation de cette inductance seule ne suffit donc pas à répondre simultanément aux objectifs de filtrage et de faible consommation de puissance réactive.

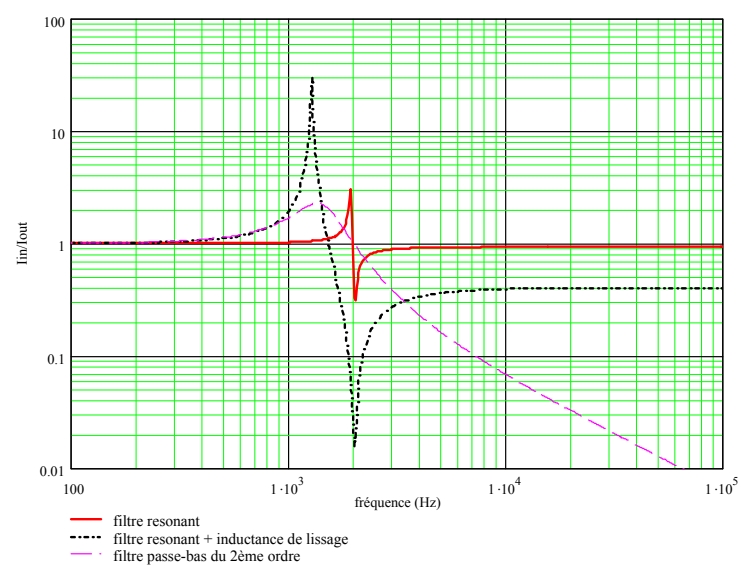

Fig. 5. Fonction de transfert I1/I2 pour différents filtres

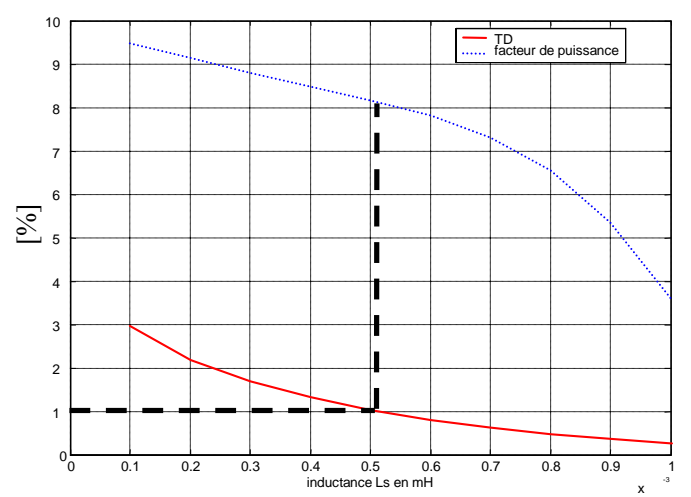

Fig. 6. TDH et facteur de puissance en fonction de l'inductance Ls $(\mathrm{P}=10 \mathrm{~kW})$

\section{Dimensionnement du filtre résonant}

On fixe une inductance série Ls de $518 \mu \mathrm{H}$, afin que le TDH soit déjà de $10 \%$. Le facteur de puissance est alors de 0.81 (Fig. 6). Un minimum de 0.9 est imposé par les normes aéronautiques. On peut alors dimensionner la capacité du filtre pour qu'elle délivre une puissance réactive de 4350VAR (ce qui ramène le facteur de puissance à 0.9 pour une puissance active absorbée de $10 \mathrm{~kW}$ ). Cette puissance doit être fournie à la pulsation du réseau $\omega_{0}$ telle que [8] :

$$
Q=3 \cdot \frac{V^{2}}{X L C}=\frac{3 \cdot V^{2}}{\left(L_{h} \cdot \omega_{0}-\frac{1}{C_{h} \cdot \omega_{0}}\right)}=\frac{3 \cdot V^{2}}{\left(\frac{\omega_{0}}{\omega_{h}{ }^{2} \cdot C_{h}}-\frac{1}{C_{h} \cdot \omega}\right.}
$$

où, $\omega_{h}$ est la pulsation de résonance du filtre $L_{h} C_{h}(i c i h=5)$ et $\mathrm{V}$ la tension simple du réseau $(115 \mathrm{~V})$.

D'autre part, on décide de caler l'atténuation maximale sur l'harmonique à éliminer (généralement 5), ce qui donne une relation reliant $\mathrm{Lh}$ et $\mathrm{Ch}$ :

$$
\mathrm{L}_{\mathrm{h}} \cdot \mathrm{C}_{\mathrm{h}} \cdot \omega_{\mathrm{h}}{ }^{2}=1
$$

Les valeurs des composants et des pulsations obtenues par cette procédure sont résumées dans le Tableau 2.
Le tracé de l'impédance du filtre (Fig. 5) montre une antirésonance (ou résonance parallèle) autour de $1300 \mathrm{~Hz}$, qui n'est pas multiple de la fréquence du réseau, ce qui est donc acceptable.

Par ailleurs, on peut également calculer le courant dévié par le filtre au fondamental.

On obtient $7 \mathrm{~A}$, ce qui est raisonnable vue la puissance du montage.

\begin{tabular}{c|c}
\hline$\omega_{0}$ en rad$/ \mathrm{s}$ & 2513 \\
\hline$\omega_{\mathrm{h}}$ en $\mathrm{rad} / \mathrm{s}$ & 1053 \\
\hline $\mathrm{L}_{\mathrm{s}}$ en $\mu \mathrm{H}$ & 518 \\
\hline $\mathrm{C}_{\mathrm{h}}$ en $\mu \mathrm{F}$ & 18 \\
\hline $\mathrm{L}_{\mathrm{h}}$ en $\mu \mathrm{H}$ & 351 \\
\hline
\end{tabular}

TABLEAU 2 :DIMENSIONNEMENT DU FILTRE RESONANT POUR P $=10 \mathrm{KW}$

\section{ASSSOCIATION ETAGE D'ENTREE-FILTRE}

Une fois le filtre dimensionné de manière théorique, il s'agit de le tester sur la structure de l'étage d'entrée retenue. Dans un premier temps, on peut vérifier qu'il correspond bien aux attentes théoriques, au moins en terme d'atténuation. Pour cela, on récupère sous Mathcad, après simulation Spice, la FFT du courant absorbé par le redresseur, avec une inductance série de $518 \mu \mathrm{H}$, mais sans le filtre parallèle: on dispose alors de la source de perturbation que l'on se dispose de filtrer.

On applique alors la fonction de transfert I1/I2, calculée au paragraphe IV.A, ce qui permet de retrouver le courant de ligne ainsi filtré, dans l'hypothèse où il ne dépend pas du filtre (hypothèse systématiquement faite dans la théorie du filtrage).

On peut alors comparer ce résultat à une simulation Spice incluant cette fois ci le filtre. Le résultat de la Fig. 7 montre clairement que si l'harmonique 5 est bien atténuée par le filtrage, quel que soit le mode de calcul envisagé, l'amplitude de l'harmonique 7 est mal évaluée par l'approche "filtrage", vis-à-vis de la simulation temporelle. Les THD sont alors évidemment différents.

On montre donc ainsi sur cette séance que, pour des étages d'entrée de ce type, la source de perturbation dépend du filtre, et que seule une approche globale, traitant à la fois filtre et convertisseur, permet de dimensionner correctement ce filtre. Ceci sera d'autant plus vrai que l'on se permettra de faire varier l'inductance de ligne Ls !

\section{Simulation Globale}

On propose de dimensionner un filtre de manière globale. Pour cela, les trois paramètres du filtre (Ls, Lh et Ch) sont laissés libres, et on va chercher à satisfaire les contraintes en terme de THD, d'amplitude harmonique par harmonique, de facteur de puissance.

De plus la formulation du problème adoptée va permettre une optimisation du filtre, sous les contraintes précédemment évoquées. Qui dit optimisation dit fonction objectif. Celle retenue est le volume global (défini comme la somme des 
volumes des trois composants)

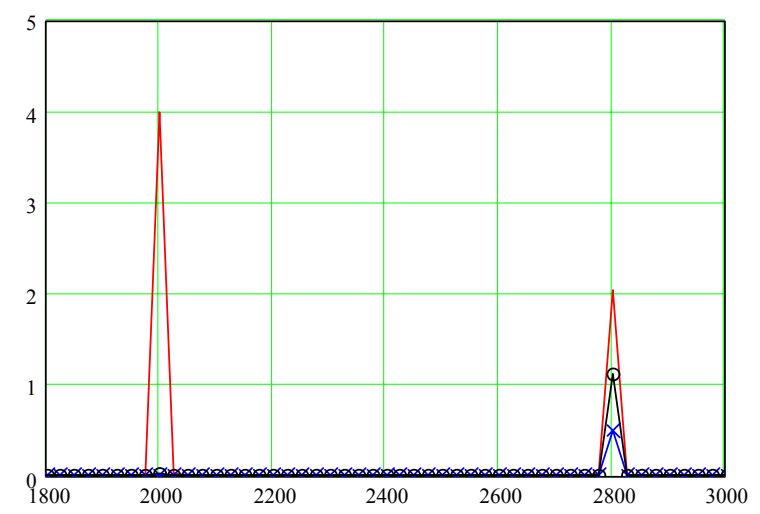

Fig. 7. Comparaison du spectre du courant ligne sans filtre (trait plein), après filtrage (hypothèse source indépendante du filtre, $\mathrm{X}$ ), et après filtrage (simulation temporelle O). Le Tableau 3 donne l'amplitude des harmoniques.

\begin{tabular}{|c|c|}
\cline { 2 - 2 } \multicolumn{1}{c|}{} & Amplitude de H7 (A) \\
\hline Pont de diode seul & 2.04 \\
\hline $\begin{array}{l}\text { Prévision filtrage (source } \\
\text { indépendante du filtre } \mathrm{X})\end{array}$ & 0.51 \\
\hline Simulation avec filtre $(\mathrm{O})$ & 1.12 \\
\hline
\end{tabular}

TABLEAU 3: AMPLITUDE DE L'HARMONIQUE 7 CORRESPONDANT A LA FIG. 7.

L'étude précédente ayant montré l'interdépendance filtreconvertisseur, il s'agit donc de simuler l'ensemble au sein de la procédure d'optimisation. La structure complète "filtre + redresseur" est simulée temporellement avec le module SimPowerSystems de Matlab et l'optimisation est réalisée avec l'opérateur ‘fmincon' du même logiciel.

\section{A. Prise en main du logiciel et vérification}

La première étape est de tester que les résultats issus du SimPowerSystems correspondent à ceux de PSpice. Ceci s'effectue sur une simulation avec et sans filtre.

\section{B. Formulation de la fonction objectif et des contraintes}

La définition de la fonction objectif retenue nécessite d'estimer le volume des éléments du filtre. Pour cela, nous avons retenu des formulations analytiques simples. Ce n'est pas réellement la précision qui compte, mais plus les sens de variation. La relation entre $C_{h}, L_{h}, L_{s}$ et leur volume dépend évidemment de la technologie utilisée. Pour les inductances, nous considérons des inductances bobinées autour d'un circuit magnétique. Dans ce cas, le volume est proportionnel à la puissance $3 / 4$ de la valeur de l'inductance [9].

$V l=K \cdot A^{3 / 4}$

Avec A égal au produit des aires Sb.Sf (respectivement surface de bobinage et surface de fer), proportionnel à l'inductance (4) et $\mathrm{K}$ une constante qui dépend des dimensions du circuit magnétique.

$\mathrm{A}=\mathrm{Sb} \cdot \mathrm{Sf}=\frac{\mathrm{Kb} \cdot \mathrm{L} \cdot \mathrm{Ieff}}{\mathrm{J} \cdot \mathrm{Bm}}$

La relation entre le volume du condensateur et sa valeur est fonction de sa permittivité, sa tension de service et sa rigidité diélectrique (respectivement $\varepsilon$, Um et E).
$\mathrm{Vc}=\frac{\mathrm{Um}^{2}}{\varepsilon \cdot \mathrm{E}^{2}} \cdot \mathrm{C}$

Avec l'hypothèse que la technologie est la même pour $\mathrm{L}_{\mathrm{s}}$ et $\mathrm{L}_{\mathrm{h}}$, la fonction objectif est donc :

Fobj $=3 \cdot \mathrm{Vc}+6 \cdot \mathrm{Vl}=3 \cdot \frac{\mathrm{Um}^{2}}{\varepsilon \cdot \mathrm{E}^{2}} \cdot \mathrm{C}+6 \cdot \mathrm{K} \cdot \mathrm{A}^{\frac{3}{4}}$

Côté contraintes, on définit des intervalles pour les valeurs de composants, et on assure la surveillance des harmoniques individuelles de courant (Tableau 1). Le taux de distorsion harmonique $(\mathrm{TDH})$ doit être inférieur à $5 \%$ et le facteur de puissance supérieur à 0.9 .

\section{Optimisation du filtrage d'un pont}

Le Tableau 4 regroupe l'ensemble des résultats après optimisation. Ils sont comparés avec ceux donnés par le dimensionnement présenté dans la partie précédente. Les résultats sont présentés pour deux puissances différentes (5 et $10 \mathrm{~kW})$.

Le faible gain en volume (1\%) amène à conclure que malgré les approximations effectuées, le dimensionnement "empirique" est très proche de l'optimum. Le tracé de la fonction de transfert du filtre (Fig. 9) après optimisation confirme que la fréquence de résonance se 'cale' naturellement vers le rang $5(1880 \mathrm{~Hz})$.

Deux séances sont réservées à cette activité. Le temps d'optimisation relativement long ne permet pas de traiter plusieurs cas, et des résultats "touts faits" sont fournis aux étudiants en fin de séance.

Des études de sensibilité sont également prévues (variation sur les éléments du filtre, sur la fréquence du réseau, sur la puissance...) pour analyser la robustesse du dimensionnement.

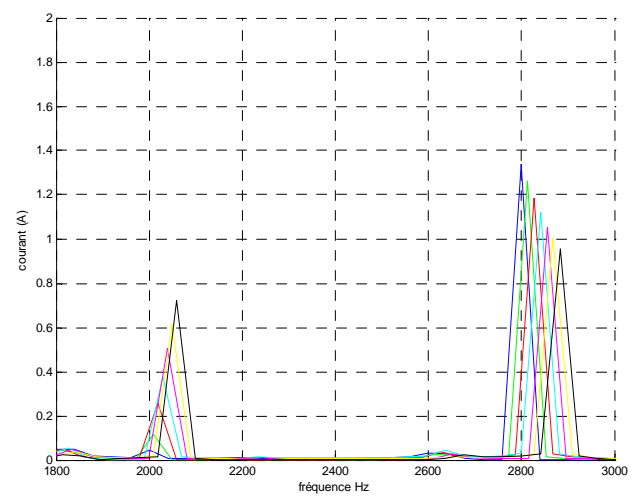

Fig. 8. Spectre du courant dans le cas d'une étude de sensibilité sur la fréquence du réseau (variation de 400 à $415 \mathrm{~Hz}$ )

\begin{tabular}{|c|c|c|c|c|}
\hline & \multicolumn{2}{|c|}{ Dimensionnement } & \multicolumn{2}{|c|}{ Optimisation } \\
\hline & $10 \mathrm{~kW}$ & $5 \mathrm{~kW}$ & $\begin{array}{c}10 \mathrm{k} \\
\mathrm{W}\end{array}$ & $5 \mathrm{~kW}$ \\
\hline $\mathrm{L}_{\mathrm{s}}(\mathrm{en} \mu \mathrm{H})$ & 518 & 1030 & 503 & 913 \\
\hline$C_{h}(e n \mu F)$ & 18 & 12 & 17 & 11.5 \\
\hline $\mathrm{L}_{\mathrm{h}}(\mathrm{en} \mu \mathrm{H})$ & 351 & 531 & 399 & 523 \\
\hline $\begin{array}{c}\text { TDH } \\
(\text { en } \%)\end{array}$ & 4.5 & 4 & 4.56 & 5 \\
\hline FP & 0.94 & 0.96 & 0.945 & 0.97 \\
\hline $\begin{array}{c}\text { Volume } \\
\text { total en } \mathrm{cm}^{3}\end{array}$ & 917 & 783 & 910 & 775 \\
\hline
\end{tabular}


TABLEAU 4 : RESULTATS APRES OPTIMISATION AVEC DEUX PUISSANCES DIFFERENTES

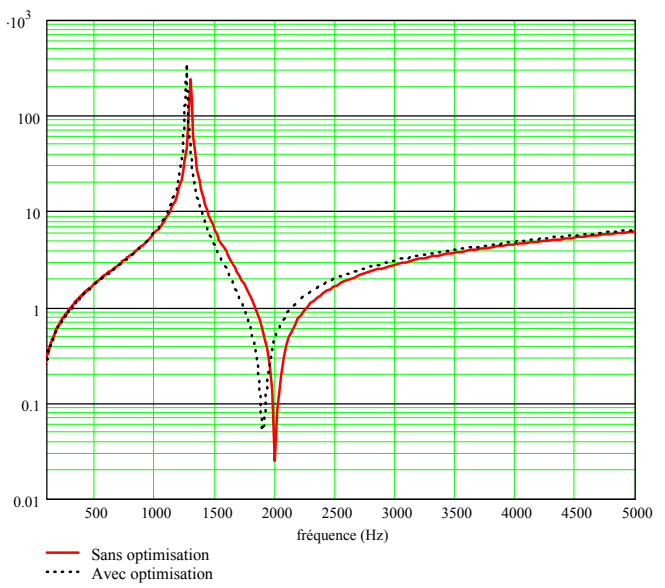

Fig. 9. Impédance du filtre (V2/I2 avec les notations de la Fig. 4) après optimisation

Ces résultats montrent cependant que la relation entre la puissance et le volume du filtre n'est pas linéaire. En terme de volume, il est préférable de dimensionner un filtre pour une puissance de $10 \mathrm{~kW}$ plutôt que deux de $5 \mathrm{~kW}$. Ceci nous amène à comparer, toujours avec la même méthode d'optimisation, quel est l'emplacement le mieux approprié pour limiter le volume du filtre en présence de deux convertisseurs de puissances identiques ou différentes.

\section{ETUDE AU SEIN D'UN "MINI-RESEAU"}

Pour vérifier cette hypothèse nous avons comparé l'optimisation dans les deux configurations de la (Fig. 10). Les ponts redresseurs sont séparés par une ligne modélisée par une inductance et sont tous deux de même puissance $(5 \mathrm{~kW})$. Les contraintes sont toujours de $5 \%$ pour le TDH et de 0.9 pour le facteur de puissance (vu de l'alternateur).

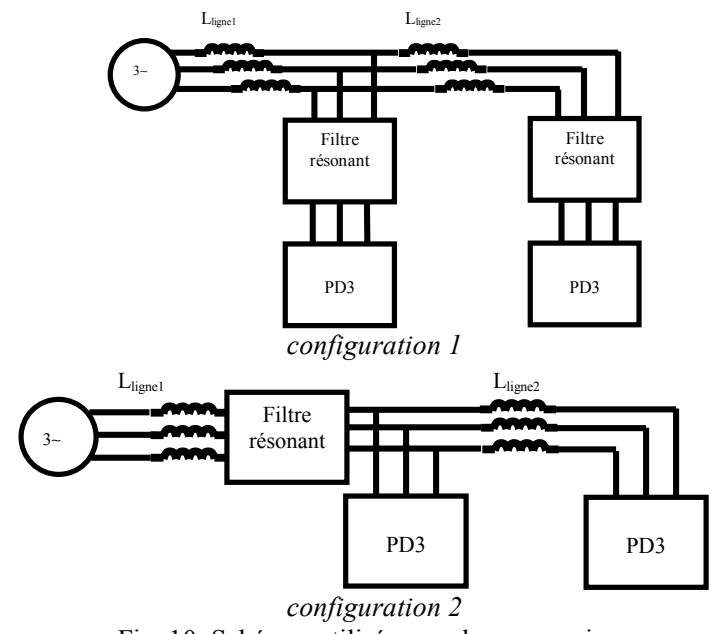

Fig. 10. Schémas utilisés pour la comparaison

Les résultats donnés dans le Tableau 5 montrent qu'il est préférable en terme de volume de faire un filtrage global plutôt qu'un filtrage pont par pont. Ce résultat ouvre des perspectives intéressantes du point de vue de la localisation optimale du filtrage d'un réseau. Dans le cas d'une installation complète, deux étapes s'avèreront nécessaires :

1. choix de la localisation optimale du (ou des) filtre(s) à l'aide d'une procédure d'optimisation. Cela pourrait être celle déjà développée dans les réseaux face au problème de plusieurs producteurs d'énergie interconnectés [10].

2. dimensionnement optimal du (ou des) filtre(s) par la méthode proposée.

\begin{tabular}{l|c|c}
\hline & Config 1 & Config 2 \\
\hline TDH en \% & 3.72 & 4.99 \\
\hline FP & 0.94 & 0.95 \\
\hline Volume en cm & $\mathbf{1 5 4 6}$ & $\mathbf{8 5 1}$ \\
\hline
\end{tabular}

Tableau 5 : Comparaison de l'optimisation dans les deux cas de figure

Dans cette séance, cette approche en terme de "mini réseau" est menée dans la même philosophie que les deux séances précédentes: les étudiants avancent leur programme Matlab le plus loin possible (implantation au sein du SimPowerSystems, vérification,...) et les résultats d'optimisation peuvent être donnés si les temps sont trop longs pour certains groupes.

\section{POUR ALLER PLUS LOIN - FILTRAGE ACTIF}

On peut également envisager des solutions alternatives au filtrage passif: des filtres actifs peuvent aisément être simulés sous Pspice, à l'aide de sources de courant. Le principe est de mesurer les harmoniques de courant issues d'une première simulation, de les sauvegarder dans un fichier texte, et de les réinjecter dans un deuxième temps (sans le fondamental !), au moyen d'une source de courant pilotée par le fichier (I_pwl sous Pspice). L'intérêt de cette analyse est d'investiguer l'influence éventuelle du point d'injection (i.e. la localisation du filtre actif).

Il est également possible d'envisager le remplacement des étages d'entrée de type "pont de diodes" par des structures de type "absorption sinusoïdale". Le coût de l'opération n'est évidemment pas anodin, mais permet d'éviter les problèmes d'harmonique basse fréquence.

Là encore, on peut analyser en simulation sous PSpice le fonctionnement d'un redresseur triphasé commandé en Modulation de Largeur d'Impulsion.

\section{CONCLUSION}

Cet article a présenté l'expérience du Master Csee à Grenoble, en terme de dimensionnement de filtre passif, sur l'exemple d'un réseau de bord d'avion. Cette étude se situe dans le cadre du projet intégrateur de ce Master professionnel. Bien évidemment, le sujet est riche, et le contenu ambitieux, mais les étudiants ont ainsi à leur disposition une large palette de centres d'intérêt potentiels. La méthodologie adoptée (utilisation d'outils variés, notion de dimensionnement global) est somme toute plus importante que les résultats chiffrés.

Le Tableau 6 illustre la répartition des séances. Des moments de cours sont prévus pour donner les grandes lignes d'utilisation des techniques informatiques (Spice, Matlab, Mathcad) et des manipulations à effectuer (copier coller de Spice vers Mathcad par exemple). Des "points bilan sont également prévus, de manière à tirer des conclusions en commun pour tous les groupes. 


\section{REFERENCES}

\begin{tabular}{|c|c|c|c|}
\hline $\begin{array}{l}\text { Séance } \\
\text { (durée) }\end{array}$ & Thème & Cours & $\begin{array}{l}\text { Point } \\
\text { bilan }\end{array}$ \\
\hline $1(2 h)$ & Présentation & $2 \mathrm{~h} 00$ & \\
\hline $2(4 h)$ & Simulation Spice & Oh30 & \\
\hline $3(4 h)$ & Filtre (Mathcad) & 0h30 & \\
\hline $4(4 h)$ & $\begin{array}{c}\text { Association filtre-CVS } \\
\text { (Spice) }\end{array}$ & Oh30 & $0 \mathrm{~h} 15$ \\
\hline $5(4 h)$ & $\begin{array}{c}\text { Simulation Matlab: } \\
\text { comparaison avec PSpice }\end{array}$ & Oh30 & \\
\hline $6(4 h)$ & $\begin{array}{c}\text { Codage des volumes et } \\
\text { fonction "fmincon" } \\
\text { d'optimisation sous } \\
\text { Matlab }\end{array}$ & $1 \mathrm{~h} 00$ & $0 \mathrm{~h} 30$ \\
\hline $7(4 h)$ & Mini réseau & & $0 \mathrm{~h} 15$ \\
\hline
\end{tabular}

[1] Power Optimised Aircraft, contract G4RD-CT-2001-00601 under the European Communities $5^{\text {th }}$ framework Programme for Research - Competitive and Sustainable Growth - Key Action ,New Perspectives in Aeronautics'

[2] L. Faleiro: «Power Optimised aircraft - the future of aircraft systems» AIAA international Air and Space Symposium: The Next 100 years

[3] K.J.Karimi, "Modeling and analysis of aircraft power system harmonics." Vol3 EPE95

[4] George J.Wakileh, "Power Systems Harmonics. Fundamentals, Analysis and filter design". DUNOD 1999

[5] S.Saadate, M.A.E. Alali «A propos des filtres passif et actif», Journées de la section Electrotechnique du club EEA, 2000

[6] Cahier technique Merlin Gernin n${ }^{\circ} 160$

[7] M. Coyaud, Caractéristaion fonctionnelle de composants en carbure de Silicium, Doctorat de l'INPG de Grenoble, juin 2002.

[8] P. Bastard, M. Meunier « Filtrage des harmoniques dans un réseau d'énergie »

[9] JP. Ferrieux F.Forest «Alimentations à découpage Convertisseurs à résonance » $3^{\mathrm{ème}}$ édition. DUNOD 1999

TABLEAU 6. REPARTITION DES SEANCES.

[10] R. Caire, N. Retière, E. Morin, M. Fontela, N. Hadjsaid «Voltage Management of Distributed Generation in Distribution Network», IEEE Power Engineering Society 2003 General Meeting, 13-17 